\title{
Helicity oscillations of Dirac and Majorana neutrinos
}

\author{
Alexandra Dobrynina, ${ }^{1,2}$ Alexander Kartavtsev, ${ }^{2}$ and Georg Raffelt ${ }^{2}$ \\ ${ }^{1}$ P. G. Demidov Yaroslavl State University, Sovietskaya 14, 150003 Yaroslavl, Russia \\ ${ }^{2}$ Max-Planck-Institut für Physik (Werner-Heisenberg-Institut), Föhringer Ring 6, 80805 München, Germany
}

\begin{abstract}
The helicity of a Dirac neutrino with mass $m$ evolves under the influence of a $B$-field because it has a magnetic dipole moment proportional to $m$. Moreover, it was recently shown that a polarized or anisotropic medium engenders the same effect for both Dirac and Majorana neutrinos. Because a $B$-field polarizes a background medium, it instigates helicity oscillations even for Majorana neutrinos unless the medium is symmetric between matter and antimatter. Motivated by these observations, we review the impact of a $B$-field and of an anisotropic or polarized medium on helicity oscillations for Dirac and Majorana neutrinos from the common perspective of in-medium dispersion.
\end{abstract}

PACS numbers: 14.60.Pq, 13.15.+g, 97.60.Bw

\section{INTRODUCTION}

The left-handed nature of weak interactions implies that astrophysical neutrino ensembles emerge far from helicity equilibrium, notably in the early universe and the interior of collapsing stars or merging neutron stars. On the other hand, the "wrong-helicity" components are not completely sterile because neutrinos have small masses. Moreover, there could be new interactions which could accelerate the relaxation toward left-right equilibrium, although we will here focus on the impact of neutrino masses alone. In the latter case, the rate towards helicity equilibrium is of order $(m / 2 E)^{2}$ times an ordinary weak rate, where $E$ is a typical neutrino energy. This rate tends to be far too small to be of practical interest. However, the helicity conversion rate can be coherently enhanced in the form of helicity oscillations.

It has long been known that nonzero neutrino masses imply magnetic and electric dipole and transition moments [1-7], allowing $B$-fields to instigate spin and spin-flavor oscillations [8-11]. Only Dirac neutrinos have intrinsic magnetic dipole moments, whereas both Dirac and Majorana neutrinos have magnetic and electric transition moments originating from the mismatch between mass and interaction eigenstates.

It was only recently fully appreciated that analogous effects arise for both Dirac and Majorana neutrinos if the background medium is anisotropic either in the form of a convective current (background vector current) or polarized (background axial vector current) [12-18]. The origin of this effect is the mismatch between chirality and helicity for neutrinos with small masses in analogy to the origin of the traditional dipole moment. A medium polarization normally originates from a $B$ field which therefore can flip the spin both by the intrinsic dipole moment and indirectly by polarizing the medium. The interaction energy of both effects must be of order $e B G_{\mathrm{F}} m$, so it is not obvious which is more important.

One way to look at this phenomenon is the perspective that in a medium both a Dirac and a Majorana neutrino acquires an effective magnetic moment: the spin polarization of the medium caused by the $B$-field is interpreted as an in-medium electromagnetic vertex function. A significant body of literature has studied this point to determine the dispersion law of active neutrinos and antineutrinos, but usually not with an eye for helicity evolution [19-31]. We here take the opposite approach and interpret even the normal $B$-field as yet another helicity-changing refractive medium and not in terms of intrinsic dipole moments.

The purpose of our short review is to look at neutrino helicity flipping by a background medium ( $B$-field, unpolarized medium with currents, polarized medium) from a common perspective and to provide explicit expressions for different cases, considering both Dirac and Majorana neutrinos with equal or different masses and considering a medium with a large matter-antimatter asymmetry as well as one which is matter-antimatter symmetric. Most of these results can be found scattered in the literature, but we hope to provide a useful clarification by unifying them from a common perspective.

We begin in Sec. II with general aspects of neutrino dispersion and discuss how anisotropic media lead to helicity conversion when neutrinos have Dirac or Majorana masses. In Sec. III we turn specifically to a $B$-field and show that the general formulas coincide with the usual description in terms of dipole moments. In Sec. IV we consider an electron gas polarized by a $B$-field and compare with the $B$-field-only case. We conclude in Sec. V.

\section{HELICITY CONVERSION}

In this section we study generic aspects of neutrino dispersion and helicity evolution in homogeneous and static media or $B$-fields on the level of refraction (forward scattering). Of course, in this setup a nontrivial evolution arises only if the initial state does not coincide with one of the in-medium propagation eigenstates.

\section{A. Neutrino dispersion}

The medium causes a shift of the neutrino self-energy $\Sigma$ by a background-induced term $\Sigma_{\mathrm{b}}$. In the Dirac equation in Fourier space, it appears in the form

$$
\left(\not k-m-\Sigma_{\mathrm{b}}\right) \psi=0,
$$

where $k$ is the neutrino four-momentum, $\not k=k_{\mu} \gamma^{\mu}$, and $m$ the neutrino mass. Generally $\Sigma_{\mathrm{b}}$ has a nontrivial Dirac struc- 
ture and depends on $k$ besides medium properties. Nontrivial solutions require $\operatorname{det}\left(\not k-m-\Sigma_{\mathrm{b}}\right)=0$, equivalent to asking for the poles of the propagator. This condition determines the dispersion relation of the propagation eigenstates in the given background medium.

The neutrino self-energy is the "blob" (the truncated matrix element) in Fig. 1. In unitary gauge to one-loop order and ignoring background neutrinos, $\Sigma_{\mathrm{b}}$ is given by the tadpole and bubble graphs of Fig. 2 which must involve the chirality projections ${ }^{1} R=\frac{1}{2}\left(1+\gamma_{5}\right)$ and $L=\frac{1}{2}\left(1-\gamma_{5}\right)$, where neutrino lines are attached. The only Dirac structure surviving the chirality projections is $\gamma^{\mu}$ or $\gamma^{\mu} \gamma_{5}$, the latter contributing a negative sign that is absorbed in the common coefficient. Therefore, on one-loop level we arrive at the most general form

$$
\Sigma_{\mathrm{b}}=-R(a \not k+\not b) L,
$$

where $a$ is a dimensionless coefficient. The overall sign follows the convention of Ref. [41]. The four-vector $b$ of dimension energy depends on the background currents and fields. It may also involve $k$, except for a term proportional to $k$ which we have explicitly separated.

For massless neutrinos, the dispersion of active and sterile components is independent. The sterile components, if they exist, are unaffected by the medium. For the active components, the coefficient $a$ modifies $\not k \rightarrow(1+a) \not k$ in the Dirac equation and one finds ${ }^{2}$

$$
k_{0}+\frac{b_{0}}{1+a}= \pm\left|\boldsymbol{k}+\frac{\boldsymbol{b}}{1+a}\right| .
$$

Henceforth we will neglect $a \ll 1$ because perturbatively it will contribute only higher-order corrections.

This result is not as simple as it looks because $b$ can depend on $k$. However, in the ultra-relativistic limit, where dispersion effects are small, we can use the unperturbed $k$ to express $b$. Even then we must worry about the sign of $k_{0}$, where negative-energy solutions of the Dirac equation represent positive-energy antiparticles with opposite momentum. Henceforth we will write the dispersion relation for positive energies for both neutrinos and antineutrinos. After expanding to linear order in $b$ we arrive at

$$
k_{0}=|\boldsymbol{k}|-\left(b_{0}-b_{\|}\right) \times \begin{cases}1 & \text { for } \nu, \\ \eta_{b} & \text { for } \bar{\nu}\end{cases}
$$

where $b_{\|} \equiv \hat{\boldsymbol{k}} \cdot \boldsymbol{b}$ and $\hat{\boldsymbol{k}}$ is a unit vector in the direction of $\boldsymbol{k}$. The component $b_{\perp}$ transverse to the neutrino momentum does not affect neutrino dispersion in linear approximation.

\footnotetext{
${ }^{1}$ In the Russian literature, $\gamma_{5}$ is defined with opposite sign and thus appears in the definitions of $R$ and $L$ with opposite sign.

${ }^{2}$ We use the metric $(+,-,-,-)$ so that $J^{0}$ and $J_{0}$ of a four-vector $J$ are the same. For typographical simplicity we usually write $J_{0}$ for the timelike component. In an expression like $J=\left(J_{0}, \boldsymbol{J}\right)$ the three-vector $\boldsymbol{J}$ refers to the contravariant space-like components, i.e., to $J^{i}$ with $i=1,2$ or 3. The Lorentz invariant product of two four-vectors is $I J=I_{\mu} J^{\mu}=$ $I_{0} J_{0}-\boldsymbol{I} \cdot \boldsymbol{J}$.
}

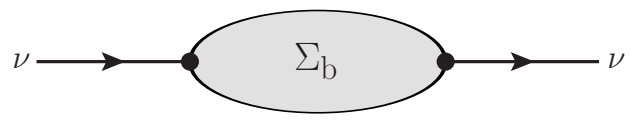

Figure 1. Neutrino self-energy graph in a background medium or electromagnetic field.
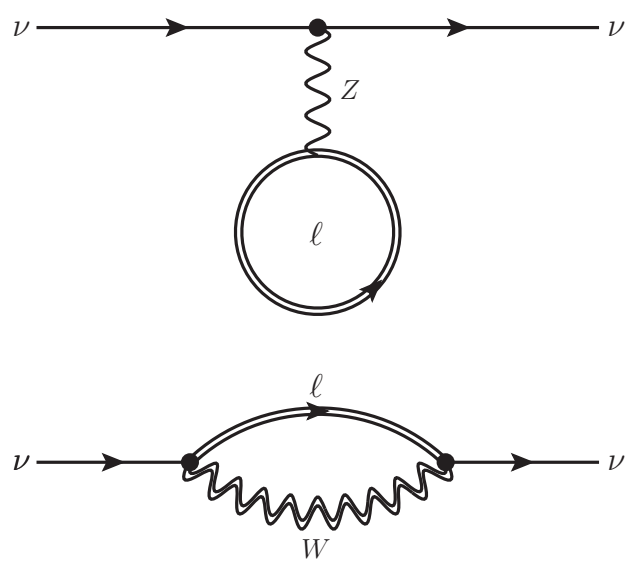

Figure 2. One-loop neutrino self-energy (unitary gauge) in a medium or B-field. Top: Tadpole graph. Bottom: Charged-current bubble graph. Double lines are dressed propagators, i.e., they include real particles of the medium and real or virtual states in the $B$-field. In our physical situations of interest, $W$ and $Z$ bosons are always virtual. We ignore background neutrinos, otherwise a $Z$ bubble would also appear. The absence of background neutrinos renders the evolution equations linear and we can study each momentum mode separately.

The parameter $\eta_{b}= \pm 1$ in Eq. (4) had to be introduced as a price for interpreting antineutrinos as positive-energy states. In particular, $\eta_{b}=-1$ when $b$ does not depend on $k$ and therefore the dispersion effect has opposite sign for $\bar{\nu}$. This is the case for an ordinary medium with large matter-antimatter asymmetry. On the other hand, $\eta_{b}=+1$ when the dispersion effect does not change sign. Typical examples are a matterantimatter symmetric medium or a $B$-field in vacuum.

Generally there are contributions to $b$ from different types of background, e.g., a medium of different components and a $B$-field. Because Eq. (4) for the ultra-relativistic limit is linear in $b$ we can consider each component separately.

\section{B. Helicity evolution}

Neutrinos have small masses so that the ultrarelativistic dispersion relation in vacuum is $k_{0}=|\boldsymbol{k}|+m^{2} / 2|\boldsymbol{k}|$. Together with flavor mixing, it leads to the usual flavor conversion effects. In addition, if $\boldsymbol{b}$ has a nonvanishing component transverse to $k$, transitions between states of opposite helicity occur to linear order in the masses. Moreover, the background effect is diagonal in the weak-interaction basis which is very different from the mass basis. Therefore, spin-flavor transitions are also possible. 

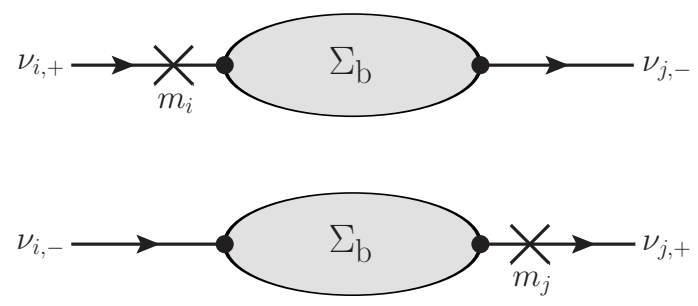

Figure 3. Spin-flip amplitudes between Dirac neutrinos with masses $m_{i}$ and $m_{j}$. The amplitude is proportional to the mass of the "wronghelicity" state attached to the blob which represents the in-medium self-energy of left-handed neutrinos.

To understand helicity conversion qualitatively, we use the mass basis and denote the eigenstates with $i$ or $j$. Without calculation we glean from Fig. 3 how the masses enter when we are in the ultrarelativistic limit. For Dirac neutrinos, which possess four degrees of freedom just like the charged leptons, only the 1.h. neutrino components communicate with the selfenergy blob, so it is the mass of the "wrong-helicity" (sterile) Dirac state which intervenes to connect to $\Sigma_{\mathrm{b}}$. In particular, the amplitude for $\nu_{i,+} \rightarrow \nu_{j,-}$ is proportional to $m_{i}$, while for $\nu_{i,-} \rightarrow \nu_{j,+}$ it is proportional to $m_{j}$. If the two masses are identical, the amplitude is proportional to $m=m_{i}=m_{j}$ and therefore the same for both cases.

To calculate the helicity-flipping amplitude it is easiest to begin with the exact spinors describing massive neutrinos in vacuum [17]. A Dirac neutrino with mass $m_{i}$, momentum $\boldsymbol{k}$, and helicity $h= \pm 1$ is described by a spinor $u_{i, h}(\boldsymbol{k})$. The forward-scattering effect of the medium is encoded in the energy shifts represented by $\Sigma_{\mathrm{b}}$. In the basis of free massive neutrinos, as opposed to the in-medium propagation eigenstates considered earlier, the shift of the neutrino Hamiltonian is

$$
\mathrm{H}_{i j, s h}^{\nu \nu}=\bar{u}_{i, s} \Sigma_{\mathrm{b}} u_{j, h},
$$

where the spinors are taken at momentum $\boldsymbol{k}$ and we use the same normalization $u_{i, h}^{\dagger} u_{i, h}=1$ as adopted in Refs. [17, 18]. A similar construction applies to antineutrinos with $\mathrm{H}_{i j, s h}^{\bar{\nu} \bar{\nu}}$ using $v$-spinors [18]. The self-energy has the simple form Eq. (2). As explained earlier, to lowest order we can restrict ourselves to the $b$ term, so that

$$
\mathrm{H}_{i j, s h}^{\nu \nu}=-\left(\bar{u}_{i, s} R\right) \not b\left(L u_{j, h}\right) .
$$

In agreement with our qualitative arguments presented earlier, $L u_{j,+} \propto m_{j}$ and $\bar{u}_{i,+} R \propto m_{i}$, so that $H_{i j,-+} \propto m_{j}$ and $H_{i j,+-} \propto m_{i}$.

Dispersion based on interaction with a medium is diagonal in the weak-interaction basis and we denote with $b_{\ell}$ the contribution related to the charged lepton $\ell$, see Fig. 2. For the transition between mass states $i$ and $j$, the relevant background charged current is therefore

$$
b_{i j}^{\mu}=\sum_{\ell=e, \mu, \tau} U_{i \ell}^{\dagger} b_{\ell}^{\mu} U_{\ell j},
$$

where $U$ is the unitary leptonic mixing matrix. Neutral-current interactions are diagonal in both the flavor and mass basis

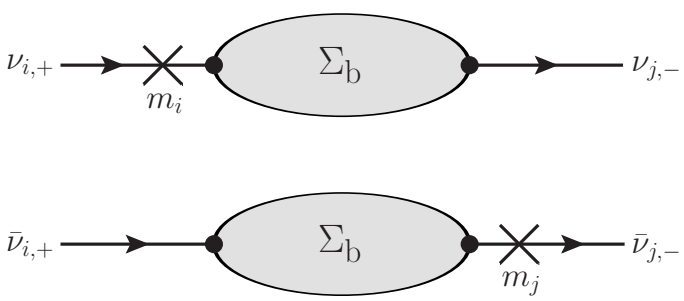

Figure 4. Spin-flip amplitudes between Majorana neutrinos with masses $m_{i}$ and $m_{j}$. The positive-helicity state can be interpreted as a "wrong-helicity" neutrino or as a "correct-helicity" antineutrino. Both amplitudes contribute with an important relative phase.

because of unitarity of the neutrino mixing matrix,

$$
\sum_{\ell=e, \mu, \tau} U_{i \ell}^{\dagger} U_{\ell j}=\delta_{i j}
$$

and therefore do not contribute to flavor or spin-flavor transitions. If neutrino-neutrino interactions are neglected then the different momentum modes decouple. Without loss of generality we may then choose a coordinate system such that for each mode the neutrino momentum is in the $z$-direction, whereas the background current lies in the $x-z$-plane. We arrange the elements of the matrix $\mathrm{H}_{i j, s h}^{\nu \nu}$ in such a way that the equation of motion can be cast in the form [18]

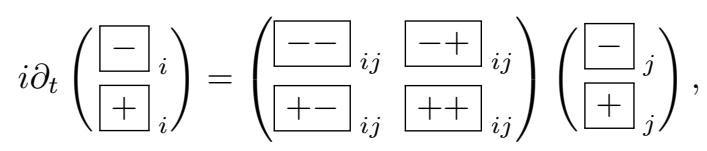

where each box denoted by helicities \pm is either a column or a $3 \times 3$ matrix in flavor space. For Dirac neutrinos we then find to linear order in neutrino masses [18]

$$
\mathbf{H}_{i j, s h}^{\nu \nu}=-\left(\begin{array}{cc}
b_{i j}^{0}-b_{i j}^{\|} & b_{i j}^{\perp} \frac{m_{j}}{2|\mathbf{k}|} \\
b_{i j}^{\perp} \frac{m_{i}}{2|\mathbf{k}|} & 0
\end{array}\right) .
$$

Indeed, the masses on the off-diagonals are the ones associated with the "wrong" (positive) helicity Dirac neutrino. For Dirac antineutrinos, a similar expression pertains for which we find [18]

$$
\mathrm{H}_{i j, s h}^{\bar{\nu} \bar{\nu}}=\eta_{b}\left(\begin{array}{cc}
0 & b_{i j}^{\perp} \frac{m_{i}}{2|\mathbf{k}|} \\
b_{i j}^{\perp} \frac{m_{j}}{2|\mathbf{k}|} & -\left(b_{i j}^{0}-b_{i j}^{\|}\right)
\end{array}\right) .
$$

Now the negative helicity is the sterile one and the masses appear accordingly. Notice also that the nonzero diagonal elements of Eqs. (10) and (11) match the energy shifts in Eq. (4), as anticipated from invariance of the eigenvalues with respect to the choice of basis. 
Majorana neutrinos have only two degrees of freedom and no helicity is wrong-both of them are active states, essentially $\nu$ and $\bar{\nu}$. Therefore, the amplitude for $\nu_{i,+} \rightarrow \nu_{j,-}$ involves both graphs in Fig. 4. For equal masses $m=m_{i}=$ $m_{j}$, the amplitude is proportional to $m-\eta_{b} m$. If the refractive effect changes sign for $\bar{\nu}$ as in an ordinary medium $\left(\eta_{b}=-1\right)$, a suitable anisotropy engenders helicity conversions. In the opposite case where $\eta_{b}=+1$ there is no helicity evolution. Typical examples are a CP-symmetric plasma or a $B$-field in vacuum, corresponding to the absence of a magnetic dipole moment for a Majorana neutrino. For unequal masses $m_{i}$ and $m_{j}$, the amplitude is proportional to $m_{i}-\eta_{b} e^{i \alpha} m_{j}$, where $\alpha$ is a relative phase which depends on the neutrino mixing matrix. Typically both Dirac and Majorana neutrinos show spinflavor oscillations in any type of anisotropic medium, and in particular, both have transition dipole moments engendered by masses and flavor mixing.

To render these qualitative arguments more quantitative, we recall that for Majorana particles $\bar{\nu}=\nu^{T} C$, where $C$ is the operator of charge conjugation. If the mean-field Hamiltonian on the level of the current-current approximation contains a term $\sum_{i j} \bar{\nu}_{i} R b_{i j} L \nu_{j}$, it can be identically rewritten as

$$
\sum_{i j} \bar{\nu}_{i} R b_{i j} L \nu_{j}=-\sum_{i j} \bar{\nu}_{i} L b_{j i} R \nu_{j}
$$

In the language of Dirac neutrinos, the $R \not b L$ structure projects out the neutrino contributions, whereas $L \not b R$ projects out the antineutrino contributions. Therefore, for Majorana neutrinos the resulting mean-field Hamiltonian can be expressed as a difference of the Dirac neutrino and antineutrino Hamiltonians in the form

$$
\mathrm{H}_{i j, s h}^{\mathrm{M}}=-\left(\begin{array}{cc}
b_{i j}^{0}-b_{i j}^{\|} & \frac{b_{i j}^{\perp} m_{j}-\eta_{b} b_{j i}^{\perp} m_{i}}{2|\mathbf{k}|} \\
\frac{m_{i} b_{i j}^{\perp}-\eta_{b} m_{j} b_{j i}^{\perp}}{2|\mathbf{k}|} & \eta_{b}\left(b_{j i}^{0}-b_{j i}^{\|}\right)
\end{array}\right)
$$

As expected, the diagonal elements again match the energy shifts of the active neutrino and antineutrino respectively.

We emphasize again that these results apply to any kind of background medium. The Dirac vs. Majorana question is the same in all cases.

\section{Unpolarized medium}

To illustrate these points let us consider the case of an ordinary medium consisting of electrons, protons and neutrons, that has been studied in detail in Refs. $[17,18]$. Neutrino dispersion is given, at low energies, by the usual currrent-current interaction. For a given background species, it leads to the dispersion effect

$$
b^{\mu}=-\sqrt{2} G_{\mathrm{F}}\left(C_{\mathrm{V}} J_{\mathrm{V}}^{\mu}-C_{\mathrm{A}} J_{\mathrm{A}}^{\mu}\right),
$$

where $C_{\mathrm{V}}$ and $C_{\mathrm{A}}$ are vector and axial-vector couplings which depend on the background species and the flavor of the test neutrino. The mean-field currents of the background fermions (field $\psi$ ) are $J_{\mathrm{V}}^{\mu}=\left\langle\bar{\psi} \gamma^{\mu} \psi\right\rangle$ and $J_{\mathrm{A}}^{\mu}=\left\langle\bar{\psi} \gamma^{\mu} \gamma_{5} \psi\right\rangle$.

In the remainder of this subsection we consider an unpolirazed medium, $J_{\mathrm{A}}=0$. If it is isotropic in its rest frame $\left(\boldsymbol{J}_{V}=0\right)$ dispersion is given in terms of the usual weak potential

$V=\sum_{e, p, n} C_{\mathrm{V}} J_{\mathrm{V}}^{0}=\sqrt{2} G_{\mathrm{F}} \times \begin{cases}\left(N_{e}-N_{\bar{e}}\right)-\frac{1}{2} N_{n} & \text { for } \nu_{e}, \\ -\frac{1}{2} N_{n} & \text { for } \nu_{\mu, \tau},\end{cases}$

where $N_{e}, N_{\bar{e}}$ and $N_{n}$ are the electron, positron and neutron number densities. As usual, the neutral-current proton and electron vector contributions cancel in a neutral medium. For antineutrinos as test particles, the potential changes sign so that $\eta_{b}=-1$.

In the laboratory frame, the medium may flow with a velocity $\boldsymbol{\beta}$ so that $b=-V(1, \boldsymbol{\beta})$. Considering a single neutrino generation with mass $m$ we find for Dirac neutrinos, antineutrinos, and Majorana neutrinos,

$$
\begin{aligned}
i \partial_{t}\left(\begin{array}{l}
\nu_{-} \\
\nu_{+}
\end{array}\right) & =V\left(\begin{array}{cc}
1-\beta_{\|} & \beta_{\perp} \frac{m}{2|\mathbf{k}|} \\
\beta_{\perp} \frac{m}{2|\mathbf{k}|} & 0
\end{array}\right)\left(\begin{array}{l}
\nu_{-} \\
\nu_{+}
\end{array}\right), \\
i \partial_{t}\left(\begin{array}{l}
\bar{\nu}_{-} \\
\bar{\nu}_{+}
\end{array}\right) & =V\left(\begin{array}{cc}
0 & \beta_{\perp} \frac{m}{2|\mathbf{k}|} \\
\beta_{\perp} \frac{m}{2|\mathbf{k}|} & -\left(1-\beta_{\|}\right)
\end{array}\right)\left(\begin{array}{l}
\bar{\nu}_{-} \\
\bar{\nu}_{+}
\end{array}\right), \\
i \partial_{t}\left(\begin{array}{l}
\nu \\
\bar{\nu}
\end{array}\right) & =V\left(\begin{array}{cc}
1-\beta_{\|} & \beta_{\perp} \frac{m}{|\mathbf{k}|} \\
\beta_{\perp} \frac{m}{|\mathbf{k}|} & -\left(1-\beta_{\|}\right)
\end{array}\right)\left(\begin{array}{l}
\nu \\
\bar{\nu}
\end{array}\right) .
\end{aligned}
$$

Note that the transverse current of an ordinary medium engenders a nontrivial helicity evolution even for Majorana neutrinos. Spin-flavor oscillations are given by similar equations with the more complicated flavor structure of Eqs. (10)-(13).

Whereas at low energies neutrinos interact with the mean field of an ordinary medium in the usual form of a currentcurrent Hamiltonian, the medium of the early universe is nearly matter-antimatter symmetric and the current-current refractive effect vanishes. Refractive effects are still engendered by the nonlocal structure of the interaction, i.e., at higher order in inverse gauge boson masses. If the medium is isotropic, the contribution of electrons and positrons to $\nu_{e}$ dispersion, see Eqs. (4), (10) and (11), is [42]

$$
b_{0}=\frac{8 \sqrt{2} G_{\mathrm{F}} k_{0}}{3 m_{W}^{2}}\left(\left\langle E_{e}\right\rangle N_{e}+\left\langle E_{\bar{e}}\right\rangle N_{\bar{e}}\right)
$$

Here, $E_{e}$ is the energy of background electrons, and $E_{\bar{e}}$ of positrons. In this example, $b_{0}$ is proportional to the neutrino energy $k_{0}$ so that the dispersion effect is the same for $\nu_{e}$ and $\bar{\nu}_{e}$, in our convention meaning that $\eta_{b}=+1$. 


\section{MAGNETIC FIELD}

As another specific case we now turn to neutrinos propagating in a $B$-field in vacuum. Usually this situation is described in terms of neutrino dipole moments induced by their masses, but we should be able to obtain the same results if we think of the $B$-field as a background medium affecting neutrinos in analogous ways as a general anisotropic medium.

\section{A. Background current}

The relevant self-energy graph at one-loop order in unitary gauge is shown in Fig. 2 (bottom). The double lines stand for the dressed propagator of the virtual particles in the external $B$-field. To linear order in $B$ and for a given charged lepton $\ell$ in the loop and neutrino four-momentum $k$ one finds $[2,3,6]$

$$
b_{\ell}^{\mu}=\frac{6 e \sqrt{2} G_{\mathrm{F}}}{(4 \pi)^{2}} f\left(r_{\ell}\right) k_{\alpha} \widetilde{F}^{\alpha \mu},
$$

where $\widetilde{F}^{\mu \nu}=\frac{1}{2} \epsilon^{\mu \nu \alpha \beta} F_{\alpha \beta}$ is the dual electromagnetic fieldstrength tensor which in the laboratory frame is assumed to be a pure $B$-field. In this frame, the Lorentz structure is explicitly

$$
k_{\alpha} \widetilde{F}^{\alpha \mu}=-\left(\boldsymbol{B} \cdot \boldsymbol{k}, k_{0} \boldsymbol{B}\right) .
$$

The charged-lepton dependent factor depends on the mass ratio $r_{\ell}=\left(m_{\ell} / m_{W}\right)^{2}$ in terms of the function

$$
f(r)=\frac{2-5 r+r^{2}}{2(1-r)^{2}}-\frac{r^{2} \ln r}{(1-r)^{3}}=1-\frac{r}{2}+\mathcal{O}\left(r^{2}\right) .
$$

For all charged leptons, $r_{\ell} \ll 1$ so that we may always use the lowest nontrivial expansion in $r_{\ell}$. Therefore, overall we find

$$
b_{\ell}=-\frac{6 e \sqrt{2} G_{\mathrm{F}}}{(4 \pi)^{2}}\left(1-\frac{r_{\ell}}{2}\right)\left(\boldsymbol{k} \cdot \boldsymbol{B}, k_{0} \boldsymbol{B}\right),
$$

which indeed has the dimension of energy.

\section{B. Helicity flip}

The components of $b^{\mu}$ include $b_{0} \propto|\boldsymbol{k}| B_{\|}, b_{\|} \propto k_{0} B_{\|}$, and $b_{\perp} \propto k_{0} B_{\perp}$. In the Hamiltonian matrices Eqs. (10)-(13), the refractive effect on the diagonal is always proportional to $b_{0}-b_{\|}$which is now proportional to $\left(|\boldsymbol{k}|-k_{0}\right) B_{\|}$and thus of order $\left(m^{2} / 2 E\right) B_{\|}$. Therefore, to linear order in neutrino masses, $B$-fields in vacuum do not produce a refractive effect for neutrinos moving parallel to $\boldsymbol{B}$.

To obtain the helicity-changing elements for equal masses $m=m_{i}=m_{j}$ we observe that $b$ is proportional to $k$, implying that the refractive effect is the same for $\nu$ and $\bar{\nu}$ and hence $\eta_{b}=+1$. Therefore, Majorana neutrinos do not suffer helicity evolution. For Dirac neutrinos, on the other hand,

$$
\mathrm{H}_{i i,+-}^{\nu \nu}=\frac{3 e \sqrt{2} G_{\mathrm{F}}}{(4 \pi)^{2}} m_{i} B_{\perp}
$$

where the multiplier of $B_{\perp}$ is recognized as the usual magnetic dipole moment for massive Dirac neutrinos. Notice that $E$ in the denominator of $m / 2 E$ has canceled against $k^{0}$ in $b_{\perp}$, so indeed the helicity-flip element does not depend on the neutrino energy.

For transitions between different mass eigenstates, the term which is independent of the charged fermion drops out because of the unitarity of the neutrino mixing matrix shown in Eq. (8). Therefore, the first nonvanishing contribution comes from the second term in the expansion Eq. (20). With

$$
F_{i j}=-\frac{1}{2} \sum_{\ell=e, \mu \tau} U_{i \ell}^{\dagger}\left(\frac{m_{\ell}}{m_{W}}\right)^{2} U_{\ell j}
$$

we thus find for Dirac neutrinos

$$
\mathrm{H}_{i j,+-}^{\nu \nu}=\frac{3 e \sqrt{2} G_{\mathrm{F}}}{(4 \pi)^{2}} m_{i} F_{i j} B_{\perp} .
$$

For the opposite helicity flip, instigated by $\mathrm{H}_{i j,-+}^{\nu \nu}$, we get the other mass $m_{j}$, i.e., of the participating sterile Dirac component. For Majorana neutrinos, the corresponding element is

$$
\mathrm{H}_{i j,+-}^{\mathrm{M}}=\frac{3 e \sqrt{2} G_{\mathrm{F}}}{(4 \pi)^{2}}\left(m_{i} F_{i j}-m_{j} F_{j i}\right) B_{\perp} .
$$

As anticipated earlier, the amplitudes of $\nu_{i} \leftrightarrow \bar{\nu}_{j}$ and $\bar{\nu}_{i} \leftrightarrow \nu_{j}$ are proportional to the two participating masses added with a relative phase which depends on the details of the complex Majorana mixing matrix. Note also that the flavor-diagonal diagonal elements of Eq. (25) automatically vanish.

\section{Dipole moments}

We have formulated the impact of a $B$-field in vacuum on neutrino propagation in the same way as general neutrino dispersion in a medium. Usually, however, the same physics is described in terms of intrinsic neutrino dipole moments which lead to spin precession in external fields. To make contact with this more common language, we notice that the Lorentz structure $k_{\alpha} \widetilde{F}^{\mu \alpha}$ for the $B$-field background current given in Eq. (18) leads to the structure $k_{\alpha} \widetilde{F}^{\mu \alpha} \gamma_{\mu} L$ for the self-energy. Using common identities for Dirac matrices we can rewrite the latter as

$$
\begin{aligned}
k_{\alpha} \widetilde{F}^{\mu \alpha} \gamma_{\mu} L= & -\frac{1}{8}\left(\not k \sigma_{\mu \nu}+\sigma_{\mu \nu} \not k\right) F^{\mu \nu} \\
& +\frac{1}{8}\left(\not k \gamma^{5} \sigma_{\mu \nu}-\gamma^{5} \sigma_{\mu \nu} \not k\right) F^{\mu \nu} .
\end{aligned}
$$

Sandwiching the rhs. of Eq. (26) between $\bar{u}_{i, s}$ and $u_{j, h}$ and using the unperturbed Dirac equation on one of the external neutrinos, one obtains the equivalent structure

$$
-\frac{1}{8}\left[\left(m_{i}+m_{j}\right) \sigma_{\mu \nu} F^{\mu \nu}+\left(m_{j}-m_{i}\right) \gamma^{5} \sigma_{\mu \nu}\right] F^{\mu \nu}
$$


Comparing this with the traditional dipole Lagrangian,

$$
\mathcal{L}=-\frac{1}{2} \bar{\psi}_{i}\left(\mu_{i j} \sigma_{\mu \nu}+i \epsilon_{i j} \gamma_{5} \sigma_{\mu \nu}\right) \psi_{j} F^{\mu \nu},
$$

we can immediately read off the usual flavor structure of the magnetic and electric dipole moments of Dirac neutrinos [3],

$$
\left.\begin{array}{c}
\mu_{i j} \\
i \epsilon_{i j}
\end{array}\right\}=\frac{m_{j} \pm m_{i}}{2} \frac{3 e \sqrt{2} G_{\mathrm{F}}}{(4 \pi)^{2}}\left(\delta_{i j}+F_{i j}\right)
$$

where the lower sign refers to $\epsilon_{i j} . F_{i j}$ was defined in Eq. (23) in terms of the lepton mixing matrix.

Turning this argument around, in the ultrarelativistic limit the $\gamma_{5}$ term in Eq. (28) simply contributes a sign, depending on the helicity of the initial neutrino. Therefore, the spin precession engendered by $B_{\perp}$ corresponds to an effective transition moment of the form $\mu_{\mathrm{eff}, i j}=\mu_{i j} \pm i \epsilon_{i j}$. With Eq. (29), this amounts to

$$
\mu_{\mathrm{eff}, i j}=\left(\frac{m_{j}+m_{i}}{2} \pm \frac{m_{j}-m_{i}}{2}\right) \frac{3 e \sqrt{2} G_{\mathrm{F}}}{(4 \pi)^{2}} F_{i j} .
$$

The signs of the masses in the magnetic and electric dipole moments work out such that the amplitude is proportional to the mass of the "wrong-helicity" neutrino participating in the spin-flavor process in agreement with our earlier discussion.

In the diagonal case $(i=j)$, the coefficients $\mu=\mu_{i i}$ and $\epsilon=\epsilon_{i i}$ are the usual magnetic and electric dipole moments. Note that the latter automatically vanishes in the one-loop approximation. In the neutrino rest frame, the nonrelativistic reduction corresponds to a Hamiltonian describing a two-level system in the form $-(\mu \boldsymbol{B}+\epsilon \boldsymbol{E}) \cdot \boldsymbol{\sigma}$, where $\boldsymbol{\sigma}$ is a vector of Pauli matrices. If the neutrino spin is not aligned with the respective electromagnetic field, it precesses around the field direction with frequency $2 \mu B$ or $2 \epsilon E$, respectively.

We now take the neutrino to be ultrarelativistic in the laboratory frame where only a $B$-field exists. One can easily derive the neutrino spin evolution by a Lorentz transformation to the rest frame, where both a magnetic and electric field appear, and then back to the laboratory frame. The well-known answer is that the longitudinal component $B_{\|}$contributes with a strength reduced by a factor of order $m / E$ caused by the Lorentz transformations and can be neglected. The transverse component $B_{\perp}$ leads to spin precession with an effective magnetic moment $\mu_{\mathrm{eff}}=\mu+i \epsilon$.

In particular, this result means that the neutrino electric dipole moment leads to a spin precession of ultrarelativistic neutrino in a background magnetic field as first noted by Okun [43]. Assuming the neutrino starts as a helicity eigenstate, the magnetic moment leads to a precession in the plane perpendicular to $\boldsymbol{B}_{\perp}$. The electric dipole moment, on the other hand, leads to a precession in the plane spanned by $\boldsymbol{B}_{\perp}$ and the neutrino velocity $\boldsymbol{v}$. In other words, the precession is around the direction $\boldsymbol{B}_{\perp} \times \boldsymbol{v}$ which is the electric field direction seen by the neutrino in its rest frame. So for ultrarelativistic neutrinos, the real part of $\mu_{\text {eff }}$ leads to a "magnetic" precession around $\boldsymbol{B}_{\perp}$, the imaginary part to an "electric" one around the direction of $B_{\perp} \times \boldsymbol{v}$.
For Majorana neutrinos, the diagonal dipole moments vanish, whereas the transition moments are [3]

$$
\begin{aligned}
\mu_{i j} & =\left(m_{i}+m_{j}\right) \frac{3 e \sqrt{2} G_{\mathrm{F}}}{(4 \pi)^{2}} i \operatorname{Im} F_{i j}, \\
i \epsilon_{i j} & =\left(m_{j}-m_{i}\right) \frac{3 e \sqrt{2} G_{\mathrm{F}}}{(4 \pi)^{2}} \operatorname{Re} F_{i j} .
\end{aligned}
$$

Notice that $F_{i j}$ is, strictly speaking, different from the Dirac case because the leptonic mixing matrix for Majorana neutrinos involves two additional Majorana phases beyond the Dirac phase. In general, $F_{i j}$ has both a real and an imaginary part. Therefore, the spin precession will involve both the magnetic and electric type in the sense explained earlier.

Overall, for both Dirac and Majorana neutrinos, the spinflavor transition $\nu_{i+} \leftrightarrow \nu_{j-}$ is proportional to $m_{i}$ whereas $\nu_{i-} \leftrightarrow \nu_{j+}$ is proportional to $m_{j}$, i.e., they are different in contrast to what is usually written in the literature on spinflavor oscillations. The reason is that spin-flavor transitions of ultarelativistic neutrinos are governed by an effective transition moment of the form $\mu_{i j} \pm i \epsilon_{i j}$, and the electric transition moment is frequently ignored in the literature.

In summary, the usual description in terms of dipole moments leads to the same spin-flavor oscillation effects as found in our earlier discussion in terms of a general background.

\section{Strong $B$-field}

The discussion so far was limited to the weak-field case where only the linear response to the applied $B$-field was considered and the effect can be lumped into a set of intrinsic neutrino magnetic and electric dipole and transition moments. However, if the $B$-field becomes very large, one needs to go beyond linear approximation in the dressed propagators of charged particles in Fig. 2.

Calculations of the neutrino self-energy under the influence of an external electromagnetic field beyond linear approximation have a long history [6, 31-40]. The probably most comprehensive study is Ref. [6]. The linear $B$-regime is appropriate for $e B<m_{W}^{2}$, i.e., below the $W$-critical field strength of $B_{W}=m_{W}^{2} / e=1.09 \times 10^{24} \mathrm{G}$. Effects quadratic in $B$ include a modification of the dipole moments and the appearance of an energy shift for massless neutrinos.

\section{E. Gauge invariance}

We have taken the attitude that the origin of neutrino masses and neutrino interactions are unrelated and that the mechanism which provides a Dirac or Majorana mass acts only on the external legs of the self-energy graph in Fig. 2. In the classic calculation of neutrino dipole moments by Shrock [3], the author uses unitary gauge and argues that physical results must be gauge invariant so that we can use any gauge that is convenient. The other classic calculation by Pal and Wolfenstein [2] was performed in 't Hooft-Feynman gauge, 


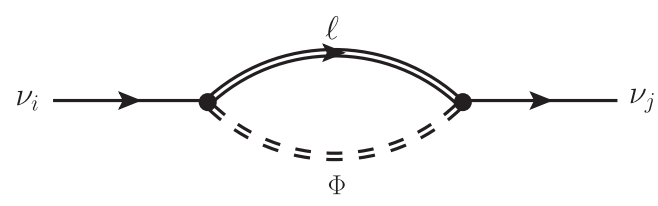

Figure 5. Contribution of the charged Higgs (in unitary gauge it becomes the longitudinal degree of freedom of the $W$-boson) to the neutrino self-energy.

where the unphysical charged scalar couples to the neutrino and charged lepton, see Fig. 5.

For Dirac neutrinos and assuming their masses arise from ordinary Yukawa couplings, gauge invariance of the magnetic dipole moment was explicitly demonstrated in Ref. [6] using the general $R_{\xi}$ gauge. In this gauge, the neutrino mass appears on the level of an interaction vertex [6],

$$
\mathcal{L}=-\frac{g}{\sqrt{2} M_{W}}\left[\left(\bar{\nu}_{i} U_{i \ell}^{\dagger} K_{i \ell} \ell\right) \Phi^{*}+\text { h.c. }\right]
$$

where $K_{i \ell}=m_{\ell} R-m_{i}^{\mathrm{D}} L$ with $m_{\ell}$ the Dirac mass of the charged lepton $\ell$. Moreover, $m_{i}^{\mathrm{D}}$ is the Dirac mass term for neutrino $i$, which coincides with the physical neutrino mass, $m_{i}^{\mathrm{D}}=m_{i}$, if the Higgs interaction is the only contribution. To linear order in $m_{i}$ the resulting contribution to the selfenergy is proportional to $m_{i} L+m_{j} R$. Sandwiching it between $\bar{u}_{i, s}$ and $u_{j, h}$ we find again that, to leading order in neutrino masses, the transition amplitude $\nu_{i,-} \rightarrow \nu_{j,+}$ is proportional to $m_{j}$, and the transition amplitude $\nu_{i,+} \rightarrow \nu_{j,-}$ is proportional to $m_{i}$. Thus, the neutrino Dirac mass is associated with the external neutrino line of the "wrong" helicity. In other words, the flavor structure of the transition amplitude produced by the diagram in Fig. 5 matches the one following from Fig. 2, which is a prerequisite for the gauge invariance of the total transition amplitude.

The propagators of the gauge and charged Higgs bosons depend on the gauge parameter $\xi$, where the limit $\xi \rightarrow \infty$ corresponds to unitary gauge, whereas $\xi=1$ corresponds to 't Hooft-Feynman gauge. The leading contribution to the self-energy, proportional to $G_{\mathrm{F}}$, is independent of the gauge choice. The next-to-leading contribution, proportional to $G_{\mathrm{F}} / M_{W}^{2}$, is given by a sum of terms, each of which is gauge dependent. However, the gauge-dependent terms cancel out in the sum and the resulting contribution to the selfenergy is gauge independent as well [6].

To perform an explicit calculation for Majorana neutrinos in an $R_{\xi}$ gauge one needs a concrete model. Pal and Wolfenstein [2] specifically used a type-II see-saw model involving a Higgs triplet. In the see-saw type-I scenario, there are no additional Higgs fields, but the right-handed neutrino acquires a large Majorana mass term $M_{\mathrm{M}}$. Diagonalizing the full mass matrix one finds that light states acquire masses of the order of $m \sim m_{\mathrm{D}}^{2} / M_{\mathrm{M}}$, while the heavy states get masses of the order of $M_{\mathrm{M}}$. For the light mass eigenstates the Lagrangian has a form identical to the one in Eq. (32), except that the Dirac mass term $m_{i}^{D}$ in $K_{i \ell}$ is replaced by the physical neutrino mass $m_{i}$ and that the $3 \times 3$ matrix $U$ is no longer unitary, but is a submatrix of the $6 \times 6$ unitary mixing matrix. This implies, in particular, that the proof of the gauge invariance of the transition amplitude in the type-I see-saw scenario reduces to that for Dirac neutrinos.

\section{POLARIZED MEDIA}

Our initial interest was triggered by the observation that a medium polarized by the $B$-field leads to helicity oscillations in addition to those caused directly by the $B$-field. One crucial insight is that particles and antiparticles with spins pointing in the same direction have the same, not opposite, refractive effect. However, they have opposite opposite magnetic moments. Therefore, an ambient $B$-field polarizes them in opposite directions and the particle and antiparticle contributions to the induced spin polarization subtract.

From Lorentz covariance considerations one expects the $B$ field induced axial-current contribution to the four-vector $b^{\mu}$ in the self-energy Eq. (2) to have the structure $u_{\alpha} \widetilde{F}^{\alpha \mu}$, where $u$ is the four velocity of the medium [23]. In the remainder of this section we assume to have a pure $B$-field in the rest frame of the medium, so the structure is $b \propto(0, \boldsymbol{B})$. This expression is to be compared with the $B$-field term in vacuum given in Eq. (19) where the role of $u$ is played by the neutrino four momentum $k$. While both terms are linear in $\boldsymbol{B}$, their different structure explains their opposite sign change when the test particle is switched to an antineutrino, as well as their very different energy dependence.

We now discuss two examples which illustrate these general points, i.e., an electron gas and a particle-antiparticle symmetric medium.

\section{A. Electron gas}

The neutron and proton magnetic dipole moments are much smaller than those of electrons, so typically the polarization effect is dominated by the electrons and positrons [26] on which we focus. The electron spin-polarization contribution to neutrino refraction is given by Eq. (14). If the axial current represents the spin-polarization induced by $\boldsymbol{B}$ it has only a spatial part $\boldsymbol{J}_{\mathrm{A}} \propto \boldsymbol{B}$ and the four-vector contributing to the self-energy in Eq. (2) has the form $b=(0, \boldsymbol{b})$ with

$$
\boldsymbol{b}=C_{\mathrm{A}} \sqrt{2} G_{\mathrm{F}} \boldsymbol{J}_{\mathrm{A}} .
$$

In a bath of electrons and for $\nu_{e}$ as test particles, $C_{\mathrm{A}}=1 / 2$, whereas for $\nu_{\mu}$ and $\nu_{\tau}$ it is $-1 / 2$. For antineutrinos as test particles, the signs reverse.

Probably the most complete derivation of the induced $\boldsymbol{J}_{\mathrm{A}}$ was provided in Ref. [26] which we have independently verified. In an external $B$-field, the electrons and positrons reside in Landau levels. Ignoring radiative corrections to the electron magnetic moment, adjacent Landau levels with opposite spin are degenerate and thus cancel in the expression for the polarization. Overall only the lowest Landau level contributes which corresponds to electrons or positrons moving along the 
$B$-field direction. One therefore finds

$$
\boldsymbol{J}_{\mathrm{A}}=\frac{e \boldsymbol{B}}{2 \pi^{2}} \int_{-\infty}^{+\infty} d p\left(\frac{1}{e^{\left(E-\mu_{e}\right) / T}+1}-\frac{1}{e^{\left(E+\mu_{e}\right) / T}+1}\right)
$$

where $e \approx 0.3028 \ldots$ is the unit electric charge, $p$ the electron or positron momentum along $\boldsymbol{B}, T$ the temperature, $\mu_{e}$ the electron chemical potential, and $E=\sqrt{m_{e}^{2}+p^{2}}$ the energy of the lowest Landau level which is independent of $B$. The first term in Eq. (34) is for electrons, the second with reversed sign and reversed chemical potential for positrons.

In the limit of vanishing chemical potential (matter-antimatter symmetric plasma), $\boldsymbol{J}_{\mathrm{A}}$ vanishes as anticipated earlier. In the opposite limit of a highly degenerate electron gas (vanishing temperature) one finds

$$
\boldsymbol{J}_{\mathrm{A}}=\frac{e \boldsymbol{B}}{\pi^{2}} \sqrt{\mu_{e}^{2}-m_{e}^{2}} .
$$

These results apply to any field strength because only the lowest Landau level contributes where the electrons move parallel to the $B$-field, but the $B$-field influences the relationship between $\mu_{e}$ and electron density. In particular, in the weak-field limit, we have $p_{\mathrm{F}}=\sqrt{\mu_{e}^{2}-m_{e}^{2}}$ with $p_{\mathrm{F}}$ the electron Fermi momentum corresponding to the electron density $n_{e}=p_{\mathrm{F}}^{3} / 3 \pi^{2}$. In this case, the induced energy shift for $\nu_{e}$ is given by $[19,26]$

$$
\mathrm{H}_{i i,--}^{\nu \nu}=-\frac{e \sqrt{2} G_{\mathrm{F}} p_{\mathrm{F}}}{2 \pi^{2}} B_{\|}
$$

For $\bar{\nu}_{e}$ the overall sign of the refractive terms reverses. For the other flavors, the sign is opposite to the $\nu_{e}$ case.

For Dirac neutrinos, the spin-flip energy, i.e., the off-diagonal element in Eq. (10) is given by

$$
\mathrm{H}_{i i,+-}^{\nu \nu}=-\frac{e \sqrt{2} G_{\mathrm{F}} p_{\mathrm{F}}}{2 \pi^{2}} \frac{m_{i}}{2 E} B_{\perp}\left|U_{e i}\right|^{2}
$$

to be compared with the expression following from the usual Dirac dipole moment in Eq. (22). Therefore, the $B$-field induced spin-flip energy mediated by the degenerate electron gas is $-\left|U_{e i}\right|^{2} 4 p_{\mathrm{F}} / 3 E_{\nu}$ times that given by the vacuum dipole moment. In an astrophysical setting, for example in a supernova core, typical neutrino energies are not much smaller than a typical electron Fermi energy. Therefore, the two contributions tend to be of similar magnitude.

For Majorana neutrinos, the electron-induced spin-flip energy is twice that of the Dirac case. It is the only contribution because the intrinsic Majorana dipole moment vanishes. Notice that for Majorana neutrinos, the spin-flip energy is proportional to $m_{i}+\eta_{b} m_{i}$ and for the spin-polarization contribution $\eta_{b}=+1$, whereas for a $B$-field in vacuum $\eta_{b}=-1$ as discussed earlier. The general transformation properties of inmedium neutrino electromagnetic vertices were first studied in Ref. [22]. It was noted that a Majorana neutrino can have a nonvanishing effective electromagnetic vertex in a medium if the latter is not symmetric between particles and antiparticles.

\section{B. Symmetric medium}

A matter-antimatter symmetric medium does not cause neutrino dispersion in the framework of the low-energy currentcurrent interaction. In particular, there is no $B$-field induced spin polarization as explained earlier [19, 24-26]. Moreover, there can not be an effective Majorana dipole moment, whereas for the Dirac case there is no objection from the general transformation properties [22]. In other words, in such a medium one has similar restrictions as in vacuum which can be seen as a CPT symmetric "medium" of virtual particles.

However, in a CPT symmetric medium of real particles, approximately realized in the early universe, neutrinos suffer non-vanishing refraction [42]. In the low-energy limit, it arises from an expansion of the gauge-boson propagator, i.e., one needs to go beyond the local four-fermion interaction model. The contribution of electrons and positrons was given in Eq. (17). The modified dispersion relation is the same for neutrinos and antineutrinos.

In addition one may include a $B$-field which affects the electrons and positrons. In the limit $m_{e} \ll T \ll m_{W}$ and $B \ll T^{2}$ one finds for the $e^{-} e^{+}$contribution to $\nu_{e}$ dispersion $[25,27,31]^{3}$

$$
b^{\mu}=-\frac{e \sqrt{2} G_{\mathrm{F}} T^{2}}{6 m_{W}^{2}}\left(\boldsymbol{k} \cdot \boldsymbol{B},-k_{0} \boldsymbol{B}\right) .
$$

It has a very similar structure to the effect of a $B$-field in vacuum of Eq. (21), except for a sign change of the spatial part. In vacuum, the time-like and longitudinal space-like parts nearly cancel so that $b_{0}-b_{\|}=\mathcal{O}\left(m^{2} / E^{2}\right)$, i.e., in vacuum a $B$ field does not cause dispersion even for active neutrinos. In the present case, on the other hand, $b_{0}$ and $b_{\|}$add up so that for both $\nu_{e}$ and $\bar{\nu}_{e}$

$$
\mathrm{H}_{i i,--}^{\nu \nu}=\frac{e \sqrt{2} G_{\mathrm{F}} T^{2}}{3 m_{W}^{2}} \boldsymbol{k} \cdot \boldsymbol{B}
$$

In addition, helicity conversion by neutrino masses occurs in the now-familiar way, observing that $\eta_{b}=+1$.

\section{CONCLUSIONS}

It was recently recognized that a material medium which is not isotropic in the laboratory frame instigates neutrino spin and spin-flavor transitions caused by nonvanishing neutrino masses. It was known for a long time that analogous effects arise from a $B$-field due to neutrino dipole and transition moments, which in the simplest case result from neutrino masses and their flavor mixing.

We have discussed these effects from the common perspective of neutrino dispersion in a mean-field background, which could be a material medium, a $B$-field, or both. If neutrinos,

\footnotetext{
${ }^{3}$ Notice that in Ref. [27] the metric $(-,+,+,+)$ is used.
} 
be they Dirac or Majorana particles, have only l.h. interactions (except for their masses), the one-loop self-energy has a very simple form where for ultrarelativistic neutrinos all dispersion effects are encoded in a single four-vector which we called $b$. It is the spatial part $\boldsymbol{b}$ which causes spin or spin-flavor conversion if it has a nonvanishing component $\boldsymbol{b}_{\perp}$ transverse to the neutrino direction of motion. The dependence on neutrino masses and mixing parameters for all cases follows from the same structure.

Dirac neutrinos suffer helicity conversion, which for them is simultaneously active-sterile conversion, for all types of anisotropic backgrounds. On the other hand, Majorana neutrinos suffer helicity conversion, which for them is simultaneously neutrino-antineutrino conversion, only if $b$ changes sign when we switch from a test $\nu$ to a $\bar{\nu}$, which is the case for an ordinary background medium. It is not true in a $B$-field, in a matter-antimatter symmetric medium, or both. It is noteworthy that, while the helicity of a single Majorana neutrino is not flipped by a $B$-field directly, it is flipped by a normal medium polarized by the $B$-field. Spin-flavor transitions arise for all types of anisotropic backgrounds for both Dirac and Majorana neutrinos. The relative behavior of Dirac and Majorana neu- trinos is analogous to the well-known structure of their dipole and transition moments.

Neutrino masses are extremely small. We do not know of a realistic astrophysical or cosmological setting where the helicity evolution of ordinary neutrinos would lead to observable consequences unless the dipole or transition moments exceed those implied by the masses. We still think that our perspective on these issues adds some conceptual clarity to the recent literature on neutrino spin evolution in anisotropic media.

\section{ACKNOWLEDGMENTS}

We acknowledge partial support by the Deutsche Forschungsgemeinschaft (DFG) under Grant No. EXC-153 (Excellence Cluster "Universe"), and by the Research Executive Agency (REA) of the European Union under Grant No. PITNGA-2011-289442 (FP7 Initial Training Network Invisibles). A.D. acknowledges financial support by the Russian Foundation for Basic Research (Project No. 16-32-00066 мол-а), and by the Dynasty Foundation.
[1] K. Fujikawa and R. Shrock, The magnetic moment of a massive neutrino and neutrino spin rotation, Phys. Rev. Lett. 45, 963 (1980).

[2] P. B. Pal and L. Wolfenstein, Radiative decays of massive neutrinos, Phys. Rev. D 25, 766 (1982).

[3] R. E. Shrock, Electromagnetic properties and decays of Dirac and Majorana neutrinos in a general class of gauge theories, Nucl. Phys. B 206, 359 (1982).

[4] J. F. Nieves, Electromagnetic properties of Majorana neutrinos, Phys. Rev. D 26, 3152 (1982).

[5] J. Schechter and J. W. F. Valle, Majorana neutrinos and magnetic fields, Phys. Rev. D 24, 1883 (1981); Erratum ibid. 25, 283 (1982).

[6] A. A. Dobrynina and N. V. Mikheev, Self-energy operator of a massive neutrino in an external magnetic field, J. Exp. Theor. Phys. 118, 54 (2014) [Zh. Eksp. Teor. Fiz. 145, 65 (2014)].

[7] C. Giunti and A. Studenikin, Neutrino electromagnetic interactions: A window to new physics, Rev. Mod. Phys. 87, 531 (2015) [arXiv:1403.6344].

[8] C. W. Werntz, 1970 (unpublished), quoted after Ref. [9].

[9] A. Cisneros, Effect of neutrino magnetic moment on solar neutrino observations, Astrophys. Space Sci. 10, 87 (1971).

[10] C.-S. Lim and W. J. Marciano, Resonant spin-flavor precession of solar and supernova neutrinos, Phys. Rev. D 37, 1368 (1988).

[11] E. K. Akhmedov, Resonance enhancement of the neutrino spin precession in matter and the solar neutrino problem, Yad. Fiz. 48, 599 (1988) [Sov. J. Nucl. Phys. 48, 382 (1988)]

[12] A. Vlasenko, G. M. Fuller and V. Cirigliano, Neutrino quantum kinetics, Phys. Rev. D 89, 105004 (2014) [arXiv:1309.2628].

[13] V. Cirigliano, G. M. Fuller and A. Vlasenko, A new spin on neutrino quantum kinetics, Phys. Lett. B 747, 27 (2015) [arXiv:1406.5558].

[14] A. Vlasenko, G. M. Fuller and V. Cirigliano, Prospects for neutrino-antineutrino transformation in astrophysical environments, arXiv:1406.6724.
[15] C. Volpe, D. Väänänen and C. Espinoza, Extended evolution equations for neutrino propagation in astrophysical and cosmological environments, Phys. Rev. D 87, 113010 (2013) [arXiv:1302.2374].

[16] D. Väänänen and C. Volpe, Linearizing neutrino evolution equations including neutrino-antineutrino pairing correlations, Phys. Rev. D 88, 065003 (2013) [arXiv:1306.6372].

[17] J. Serreau and C. Volpe, Neutrino-antineutrino correlations in dense anisotropic media, Phys. Rev. D 90, 125040 (2014) [arXiv:1409.3591].

[18] A. Kartavtsev, G. Raffelt and H. Vogel, Neutrino propagation in media: Flavor, helicity, and pair correlations, Phys. Rev. D 91, 125020 (2015) [arXiv:1504.03230].

[19] V. B. Semikoz, Induced magnetic moment of the neutrino in a dispersive medium, Yad. Fiz. 46, 1592 (1987) [Sov. J. Nucl. Phys. 46, 946 (1987)].

[20] V. B. Semikoz and Y. A. Smorodinsky, Multipole electromagnetic moments of a neutrino in a dispersive medium, Zh. Eksp. Teor. Fiz. 95, 35 (1989) [Sov. Phys. JETP 68, 20 (1989)].

[21] J. F. Nieves, Neutrinos in a medium, Phys. Rev. D 40, 866 (1989).

[22] J. F. Nieves and P. B. Pal, Electromagnetic properties of neutrinos in a medium, Phys. Rev. D 40, 1693 (1989).

[23] J. C. D’Olivo, J. F. Nieves and P. B. Pal, Electromagnetic properties of neutrinos in a background of electrons, Phys. Rev. D 40, 3679 (1989).

[24] V. B. Semikoz and J. W. F. Valle, Nucleosynthesis constraints on active sterile-neutrino conversions in the early universe with random magnetic field, Nucl. Phys. B 425, 651 (1994) [hepph/9402332]; Erratum ibid. 485, 545 (1997).

[25] P. Elmfors, D. Grasso and G. Raffelt, Neutrino dispersion in magnetized media and spin oscillations in the early universe, Nucl. Phys. B 479, 3 (1996) [hep-ph/9605250].

[26] H. Nunokawa, V. B. Semikoz, A. Yu. Smirnov and J. W. F. Valle, "Neutrino conversions in a polarized medium," 
Nucl. Phys. B 501, 17 (1997) [hep-ph/9701420].

[27] A. Erdas, C. W. Kim and T. H. Lee, Neutrino selfenergy and dispersion in a medium with magnetic field, Phys. Rev. D 58, 085016 (1998) [hep-ph/9804318].

[28] A. M. Egorov, A. E. Lobanov and A. I. Studenikin, Neutrino oscillations in electromagnetic fields, Phys. Lett. B 491, 137 (2000) [hep-ph/9910476].

[29] A. Grigoriev, A. Lobanov and A. Studenikin, Effect of matter motion and polarization in neutrino flavor oscillations, Phys. Lett. B 535, 187 (2002) [hep-ph/0202276].

[30] A. I. Studenikin, Neutrinos in electromagnetic fields and moving media, Phys. Atom. Nucl. 67, 993 (2004) [Yad. Fiz. 67, 1014 (2004)].

[31] A. V. Kuznetsov, N. V. Mikheev, G. G. Raffelt and L. A. Vassilevskaya, Neutrino dispersion in external magnetic fields, Phys. Rev. D 73, 023001 (2006) Erratum: ibid. 73, 029903 (2006) [hep-ph/0505092].

[32] G. Mckeon, Propagation of a neutrino in a homogeneous magnetic field, Phys. Rev. D 24, 2744 (1981).

[33] A. V. Borisov, V. C. Zhukovsky, A. V. Kurilin and A. I. Ternov, Radiative corrections to the mass of the neutrino in an external electromagnetic field, Yad. Fiz. 41, 743 (1985) [Sov. J. Nucl. Phys. 41, 473 (1985)].

[34] A. Erdas and G. Feldman, Magnetic field effects on lagrangians and neutrino selfenergies in the Salam-Weinberg theory in arbitrary gauges, Nucl. Phys. B 343, 597 (1990).

[35] A. V. Kuznetsov and N. V. Mikheev, Neutrino dispersion properties in an external magnetic field, Phys. Atom. Nucl. 70, 1258
(2007) [Yad. Fiz. 70, 1299 (2007)].

[36] K. Bhattacharya and S. Sahu, Neutrino absorption by W production in the presence of a magnetic field, Eur. Phys. J. C 62, 481 (2009) [arXiv:0811.1692].

[37] A. Erdas, Neutrino self-energy in external magnetic field, Phys. Rev. D 80 (2009) 113004 [arXiv:0908.4297].

[38] A. V. Kuznetsov, N. V. Mikheev and A. V. Serghienko, High energy neutrino absorption by $\mathrm{W}$ production in a strong magnetic field, Phys. Lett. B 690, 386 (2010) [arXiv:1002.3804].

[39] A. Bravo Garcia, K. Bhattacharya and S. Sahu, The neutrino self-energy in a magnetized medium, Mod. Phys. Lett. A 23, 2771 (2008) [arXiv:0706.3921].

[40] P. A. Eminov, Spin and dispersion of a massive Dirac neutrino in a magnetized plasma, Zh. Eksp. Teor. Fiz. 149, 76 (2016) [J. Exp. Theor. Phys. 122, 63 (2016)].

[41] H. A. Weldon, Covariant calculations at finite temperature: The relativistic plasma, Phys. Rev. D 26, 1394 (1982).

[42] D. Nötzold and G. Raffelt, Neutrino dispersion at finite temperature and density, Nucl. Phys. B 307, 924 (1988).

[43] L. B. Okun, On the electric dipole moment of neutrino, Yad. Fiz. 44, 847 (1986) [Sov. J. Nucl. Phys. 44, 546 (1986)].

[44] K. Kiers and N. Weiss, Coherent neutrino interactions in a dense medium, Phys. Rev. D 56, 5776 (1997) [hepph/9704346]. 